\title{
Reactive oxygen species induce neurite degeneration before induction of cell death
}

\author{
Koji Fukui ${ }^{1, *}$ \\ ${ }^{1}$ Molecular Cell Biology Laboratory, Department of Bioscience and Engineering, College of Systems Engineering and Sciences, \\ Shibaura Institute of Technology, 307 Fukasaku, Minuma-ku, Saitama 337-8570, Japan
}

(Received 23 March, 2016; Accepted 25 April, 2016; Published online 6 August, 2016)

\begin{abstract}
Reactive oxygen species (ROS) induce neuronal cell death in a timeand concentration-dependent manner. Treatment of cultured cells with a low concentration of hydrogen peroxide induces neurite degeneration, but not cell death. Neurites (axons and dendrites) are vulnerable to ROS. Neurite degeneration (shrinkage, accumulation, and fragmentation) has been found in neurodegenerative disorders, such as Alzheimer's disease, Parkinson's disease, and Huntington's disease. However, the mechanism of ROS-related neurite degeneration is not fully understood. Many studies have demonstrated the relationship between mitochondrial dysfunction and microtubule destabilization. These dysfunctions are deeply related to changes in calcium homeostasis and ROS production in neurites. Treatment with antioxidant substances, such as vitamin $\mathrm{E}$, prevents neurite degeneration in cultured cells. This review describes the possibility that ROS induces neurite degeneration before the induction of cell death.
\end{abstract}

Key Words: ROS, neurite degeneration, neuron, mitochondria, calcium

\section{Reactive Oxygen Species Induce Neurite Degeneration}

Many organs are susceptible to damage by reactive oxygen species (ROS).$^{(1,2)}$ Cellular dysfunction is induced by the accumulation of the products of oxidative damage, such as proteins, ${ }^{(3)}$ phospholipids and DNA..$^{(4,5)}$ Neurons are particularly well known to be vulnerable to ROS-induced damage. There are five main reasons for this. Firstly, the ratio of total oxygen consumption in the brain is about $20 \%$, which is higher than most other organs. ${ }^{(6)}$ Secondary, polyunsaturated fatty acids (PUFAs), such as docosahexaenoic acid and eicosapentaenoic acid are present in cell membranes of the brain. ${ }^{(7,8)}$ These PUFAs are easily oxidized compared to other fatty acids. ${ }^{(9)}$ Thirdly, there are a lot of mitochondria in the brain, as the brain requires large amounts of adenosine triphosphates. Cells that have high energy requirements, such as liver cells, cardiomyocytes, and sperms cells, typically contain many mitochondria. Fourthly, it has been demonstrated that there is a relationship between transition elements and ROS production. ${ }^{(10)}$ Notably, the substantia nigra contains relatively large amounts of transition elements compared to other tissues, including other brain regions. Aluminum, mercury, and iron are catalysts for ROS generation, and the Fenton reaction is a typical example of this. Finally, antioxidant enzyme activities such as those for super oxide dismutase (SOD), catalase and glutathione peroxidase, are relatively low in the brain compared to other organs. ${ }^{(11)}$

The consequence of severe oxidative damage in neurons is cell death. ${ }^{(12-14)}$ Treatment of cultured cells with hydrogen peroxide induces cell death in a concentration- and time-dependent manner. ${ }^{(12,14)}$ Therefore, hydrogen peroxide is used commonly in experiments to model oxidative stress in vitro. Due to differences in experimental conditions, it is often difficult to accurately deter- mine the effects of hydrogen peroxide-induced oxidative damage on cells. Some researchers have used very high concentration of hydrogen peroxide, often in excess of high micromolar and low millimolar concentrations. In these cases, hydrogen peroxide may produce acute toxicity in cultured neurons. In order to determine the more subtle effects of hydrogen peroxide-induced oxidative damage in cultured cells, treatment with low hydrogen peroxide concentrations may be more effective. Hydrogen peroxide is usually produced at low levels by the SOD reaction in vivo. ${ }^{(10)}$ Interestingly, hydrogen peroxide and other kinds of ROS can act as second messengers in cells. ${ }^{(15-17)}$ Treatment of cells with low concentrations of hydrogen peroxide reflects normal in vivo condition.

Several lines of evidence have demonstrated that treatment with low concentrations of hydrogen peroxide induces neuronal dysfunction, but not cell death. ${ }^{(18-20)}$ Gardner and colleagues reported that treatment with high concentrations of hydrogen peroxide $(>10 \mathrm{mM})$ induced apoptosis which is characterized by DNA laddering. ${ }^{(18)}$ However, treatment with a low concentration of hydrogen peroxide $(0.1 \mathrm{mM})$ induced delayed cytotoxicity, but did not induce DNA ladder formation. These authors highlighted the possible protective effects of bcl-2. Chen and Ames reported that treatment with less than $100 \mu \mathrm{M}$ hydrogen peroxide did not affect protein synthesis in human diploid foreskin fibroblast (F65) cells. ${ }^{(19)}$ Although treatment with $100 \mu \mathrm{M}$ hydrogen peroxide led to an $80 \%$ depletion of nicotinamide adenine dinucleotide (NAD), less than $50 \mu \mathrm{M}$ hydrogen peroxide inhibited NAD depletion in murine macrophage (P388D1) cells. ${ }^{(20)}$ In our previous study, treatment of neuro2a and granule cells with low concentrations of hydrogen peroxide induced neurite degeneration, but not cell death. ${ }^{(12,21,22)}$ We found morphological neurite alterations, described as bead formation, ${ }^{(12,22,23)}$ and the number and density of dendrites significantly decreased in hydrogen peroxide-treated granule cells (Fig. 1). ${ }^{(21)}$ Several lines of evidence have demonstrated the induction of neurite alterations by endogenous and exogenous of hydrogen peroxide. ${ }^{(24-26)}$ These studies indicate the possibility that low concentrations of hydrogen peroxide can induce neurite degeneration prior to the induction of cell death. The next section describes the cause of ROS-induced neurite degeneration.

ROS Treatment Induces Membrane Oxidation, Microtubule Destabilization and Mitochondria Dysfunction in Axons

There are three possible mechanisms of ROS-induced neurite degeneration (Fig. 2). First, ROS can easily oxidize PUFAs in neuronal cell membranes, and enhancement of the ratio of oxidized PUFAs induces membrane curing. ${ }^{(9)}$ Many studies have

*To whom correspondence should be addressed.

E-mail: koji@sic.shibaura-it.ac.jp 


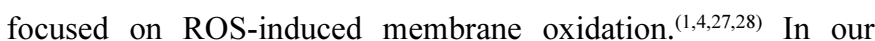
previous study, treatment with 2,2'-azobis(2-amidinopropane) dihydrochloride, which is a water-soluble free radical initiator, induced neurite beading in neuro 2 a cells. ${ }^{(22)}$ Treatment of granule cells with a low concentration of hydrogen peroxide also induced neurite degeneration, and significantly increased lipid hydroperoxide. ${ }^{(2)}$ Treatment with tocotrienols, which are a class of vitamin E, prevented hydrogen peroxide-induced neurite degeneration. ${ }^{(12,21)}$ Oxidation of membrane components, including ion channels and receptors, leads to decreased membrane permeability. For example, $N$-methyl-D-aspartate and $\alpha$-amino-3hydroxy-5-methyl-4-isoxazolepropionic-acid receptors can be damaged by ROS, which increases the influx of inorganic ions such as calcium and sodium. ${ }^{(29-31)}$ The influx of calcium ions enhances ROS production and calcium leakage in the mitochondria and endoplasmic reticulum (ER). ${ }^{(29,32)}$ These reactions further accelerate the oxidation process in cells.

The second mechanism is through the disruption of cytoskeletal proteins in the axonal region. Microtubules consist of heterodimers of alpha- and beta-tubulin, and play a crucial role in the maintenance of axonal morphology. ${ }^{(33,34)}$ Microtubule formation is typically maintained through the balance of polymerization and depolymerization of tubulins. Furthermore, microtubules play pivotal roles in cellular processes, such as a rail function in axonal

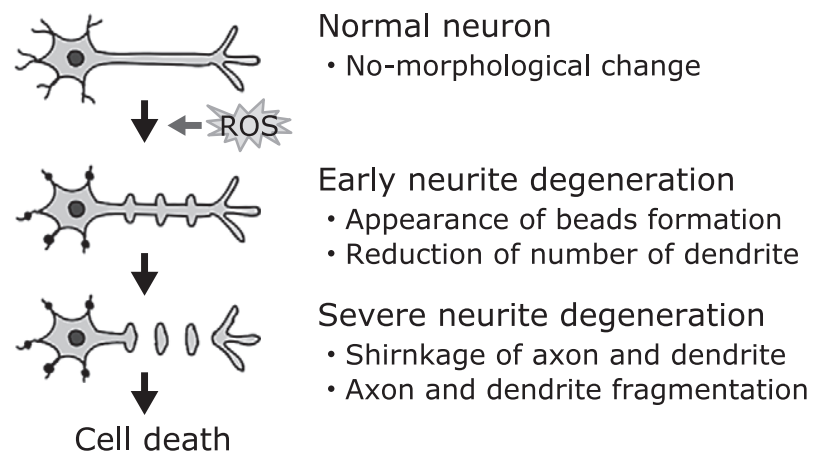

Fig. 1. Schematic of morphological changes observed in ROS-induced neurite degeneration. This schematic is based on a subset of the references cited in this review. transport. ${ }^{(23,35-37)}$ Protein synthesis and proteolysis are not possible at the distal end of axons, and many substances are moved by anterograde and retrograde transport in the axon. ${ }^{(38)}$ Microtubulerelated axonal degeneration is divided into two types. ${ }^{(23,39)}$ The first is anterograde degeneration, which involves degeneration proceeding from the cell soma to the distal end of a neurite. The second type is retrograde degeneration, which is also known as a "dying-back" process. Lunn et al. ${ }^{(40)}$ identified a mouse strain that was later termed Wallerian degeneration slow (Wlds). These mice do not exhibit axonal degeneration even 2 weeks after axonal transection. Because the Wlds mutant mouse has shown resistance to the dying-back process, this mutant model has been used to study axonal degeneration. It has been found that nicotinamide mononucleotide adenyltransferase (Nmnat) 1 and 2 protein expression protects against ROS-induced axonal degeneration in the Wlds mutant mice. ${ }^{(37,41)}$ Nmnat is related to NAD synthesis and is located in the mitochondrial matrix. These studies indicate that the maintenance of mitochondrial function may be important for maintaining neurite function. Collapsin response mediator protein (CRMP)-2 plays a pivotal role in the maintenance of microtubule stabilization. ${ }^{(42)}$ The function of CRMP-2 is very similar to tau protein, which is famously involved in the pathogenesis of Alzheimer's disease (AD). ${ }^{(43)}$ Excessive phosphorylation of CRMP-2 induces axonal dysfunction in cultured hippocampal neurons. ${ }^{(44)}$ CRMP-2 is phosphorylated by glycogen synthesis-3 beta (GSK-3 $\beta$ ) and cyclin dependent kinase 5. ${ }^{(39)}$ Calpain is a protease that is activated in the presence of calcium ions and can cleave CRMP-2. ${ }^{(4)}$ In our previous study, the ratio of CRMP-2 phosphorylation was remarkably increased in the cerebellum and hippocampus of young vitamin E-deficient and normal aged mice compared to age-matched controls. ${ }^{(46)}$ Axonal degeneration was observed following immunohistochemical analysis of hippocampal slices from vitamin E-deficient and aged mice. $^{(47)}$ Recently, the modification of tubulin (acetylation and nitration) has been identified. Because acetylated tubulin is enriched in stable microtubules, activation of histone deacetylase 5 (HDAC5), which is activated by calcium ions, accelerates microtubule depolymerization through tubulin deacetylation. ${ }^{(48,49)}$ Nitrated alpha-tubulin levels are correlated with microtubule stabilization in PC12 cells. ${ }^{(50)}$ Although these studies, including our previous reports, implicate ROS-derived microtubule dysfunction in axonal degeneration, a detailed correlation between ROS levels and microtubule disruption is not fully understood.

The third mechanism is through the disruption of the axonal transport system. Dysfunction of the axonal transport system leads to the gradual accumulation of vesicles and organelles in the axon,

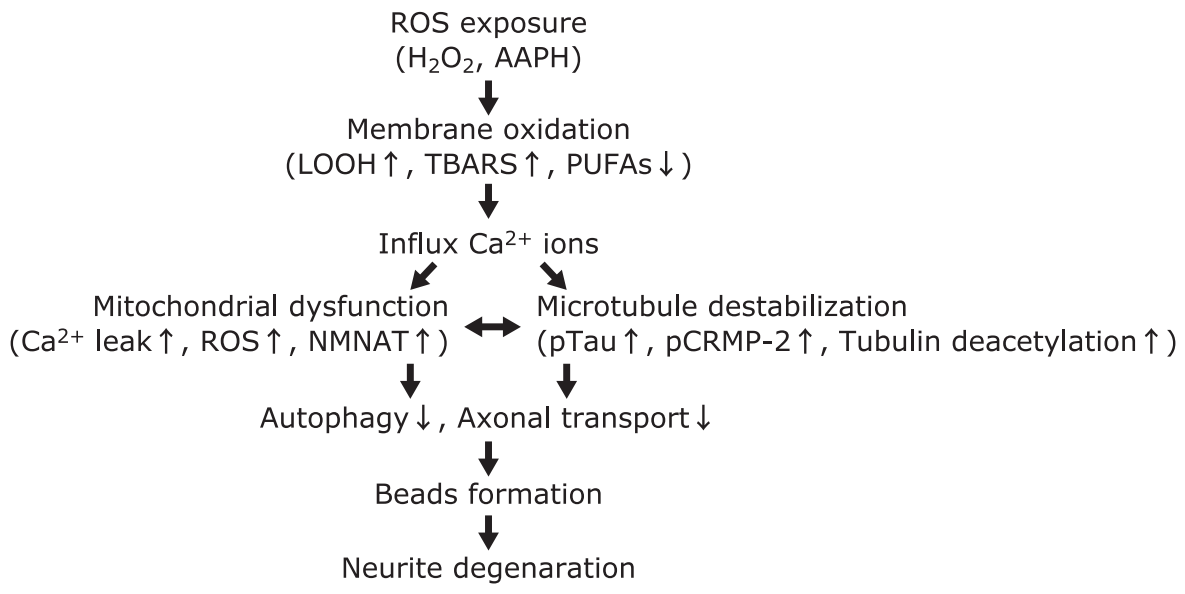

Fig. 2. Flow chart of ROS-induced neurite degeneration. This flow chart is based on a subset of references cited in this review. 
and the induction of abnormal morphology. ${ }^{(51,52)}$ In our previous study, microtubule associated protein-light chain 3 (MAP-LC3) II which is a hallmark of autophagy, was decreased in long-term vitamin E-deficient mouse brain. ${ }^{(46)}$ Several lines of evidence have demonstrated the relationship between autophagy and ROS. ${ }^{(53,54)}$ In mammalian cells, axons are rich in mitochondria, and mitochondria-induced ROS plays a role as an activator of mitophagy. ${ }^{(53)}$ ER-located bcl-2 plays a pivotal role in autophagy and is regulated by calcium ions. ${ }^{(54)}$ Changes in the axonal transport system affect normal mitochondria and organelles in that region of the cell. Finally, mitochondrial dysfunction is induced, and leakage of calcium ions and ROS production may occur. The next section describes ROS-related neurite degeneration and neurodegenerative disorders.

\section{Does ROS Induce Neurodegenerative Disorders through Neurite Degeneration?}

Several lines of evidence have demonstrated that axonal degeneration is observed in neurodegenerative disorders, such as AD, ${ }^{(23,51,55-57)}$ Parkinson's disease (PD) ${ }^{(34,35,39)}$ Huntington's disease (HD) and amyotrophic lateral sclerosis. ${ }^{(17,32,41,52,58-60)}$ Neurite degeneration is induced in AD-transgenic mice, and reductions in the motor protein kinesin-1 accelerate amyloid deposition. ${ }^{(51)}$ Induction of paired helical filaments produces axonal transport dysfunction in $\mathrm{AD} .{ }^{(55)}$ Phosphorylation of CRMP-2 blocks its ability to bind microtubules, and has been found in the early stages of AD. ${ }^{(56)}$ Treatment with beta amyloid induces neurite degeneration in SH-SY5Y cells through CRMP-2 phosphorylation. ${ }^{(57)}$ We previously reported that vitamin E-deficient mice exhibit increased phospho-CRMP-2 expression ${ }^{(46)}$ and axonal alterations in hippocampal slices. ${ }^{(47)}$ Cognitive dysfunction is the characteristic symptom of $\mathrm{AD}$, and ROS-related cognitive dysfunction may be related to neurite degeneration.

Axonal degeneration was observed in cultured dopaminergic neurons isolated from 1-methyl-4-phenylpyridiniumion $\left(\mathrm{MPP}^{+}\right)$treated C57Bl pregnant mice. ${ }^{(39)}$ The Akt/GSK-3 $\beta /$ CRMP-2 pathway is involved in this model of axonal degeneration. Treatment with $\mathrm{MPP}^{+}$induces $\mathrm{Ca}^{2+}$ release from mitochondria in isolated liver mitochondria of female Wistar rats. ${ }^{(61)}$ Microtubule polymerization is reduced in $\mathrm{MPP}^{+}$treated calf brain tubulin. ${ }^{(35)}$ Alim et $a l{ }^{(34)}$ reported that $\alpha$-synuclein, which is a common pathogenic molecule in PD, interacts with tubulin. In the PD brain, the interaction between $\alpha$-synuclein and tubulin accelerates $\alpha$ synuclein aggregation. 6-Hydroxydopamine (6-OHDA) induces axonal degeneration in dopaminergic neurons, and the mechanism is related to the induction of mitochondrial transport dysfunction, microtubule disruption, and autophagy. ${ }^{(62)}$ Treatment with antioxidant substances, such as $N$-acetyl-cystein, attenuates mitochondrial transport dysfunction. Treatment with 6-OHDA inhibits mitochondrial NADH dehydrogenase (complex 1) activity in isolated rat brain mitochondria. ${ }^{(63)}$

\section{References}

1 Butterfield DA. The 2013 SFRBM discovery award: selected discoveries from the butterfield laboratory of oxidative stress and its sequela in brain in cognitive disorders exemplified by Alzheimer disease and chemotherapy induced cognitive impairment. Free Radic Biol Med 2014; 74: 157-174.

2 Seet RC, Lee CY, Lim EC, et al. Oxidative damage in Parkinson disease: measurement using accurate biomarkers. Free Radic Biol Med 2010; 48: 560566.

3 Aoyama H, Muramoto K, Shinzawa-Itoh K, et al. A peroxide bridge between $\mathrm{Fe}$ and $\mathrm{Cu}$ ions in the $\mathrm{O} 2$ reduction site of fully oxidized cytochrome $\mathrm{c}$ oxidase could suppress the proton pump. Proc Natl Acad Sci US A 2009; 106: 2165-2169.

4 Niki E. Biomarkers of lipid peroxidation in clinical material. Biochim Biophys Acta 2014; 1840: 809-817.
ROS-induced damage has been detected in HD patients and the striatum of an HD mouse model via impairment of the mitochondrial tricarboxylic acid-cycle enzyme aconitase. ${ }^{(17)}$ Bogdanov et $a l .{ }^{(58)}$ reported that treatment with the mitochondrial toxin 3nitropropionic acid induced hydroxyl radicals in HD transgenic mice. They emphasized that mitochondria from HD transgenic mice are vulnerable to ROS. Furthermore, striatal neurons from HD transgenic mice undergo neurite degeneration via calcium leakage resultant from alteration in ER ryanodine receptors, ${ }^{(32)}$ and the accumulation of huntingtin aggregates. ${ }^{(52)}$ Huntingtin aggregates block axonal transport. Treatment with insulin and insulin like growth factor-1 reduce mitochondrial ROS production through the PI3K/Akt pathway in striatal cells from mutant HD mice. ${ }^{(59)}$

\section{Conclusion}

Neurodegenerative disorders induce axonal degeneration, and the development and progression of many neurodegenerative disorders are correlated with microtubule and mitochondrial dysfunction. Furthermore, microtubule and mitochondrial dysfunction are induced by changes in calcium homeostasis and ROS production. Treatment with antioxidant substances, such as vitamins and polyphenols, decreases the risks of the development and progression of neurodegenerative disorders, ${ }^{(64,65)}$ and inhibits axonal degeneration. ${ }^{(12,21,22)}$ Axons are highly elastic, and are more vulnerable to pathogenic compared to the cell soma, such as changes in the ion composition and increasing of ROS concentration of extracellular fluid. Axonal degeneration may be an early sign of ROS-induced neuronal degeneration.

\section{Abbreviations}

$\begin{array}{ll}\text { AD } & \begin{array}{l}\text { Alzheimer's disease } \\ \text { CRMP }\end{array} \\ \text { collapsin response mediator protein } \\ \text { glycogen synthesis-3 beta }\end{array}$

\section{Conflict of Interest}

No potential conflicts of interest were disclosed.

5 Fraga CG, Shigenaga MK, Park JW, Degan P, Ames BN. Oxidative damage to DNA during aging: 8-hydroxy-2'-deoxyguanosine in rat organ DNA and urine. Proc Natl Acad Sci U S A 1990; 87: 4533-4537.

6 Reiter RJ. Oxidative damage in the central nervous system: protection by melatonin. Prog Neurobiol 1998; 56: 359-384.

7 Watson SN, Nelson MA, Wildering WC. Redox agents modulate neuronal activity and reproduce physiological aspects of neuronal aging. Neurobiol Aging 2012; 33: 149-161.

8 Sies H. Oxidative stress: oxidants and antioxidants. Exp Physiol 1997; 82 291-295.

9 Omoi NO, Arai M, Saito M, et al. Influence of oxidative stress on fusion of pre-synaptic plasma membranes of the rat brain with phosphatidyl choline liposomes, and protective effect of vitamin E. J Nutr Sci Vitaminol (Tokyo) 
2006; 52: 248-255.

10 Markesbery WR. Oxidative stress hypothesis in Alzheimer's disease. Free Radic Biol Med 1997; 23: 134-147.

11 Urano S, Asai Y, Makabe S, et al. Oxidative injury of synapse and alteration of antioxidative defense systems in rats, and its prevention by vitamin E. Eur J Biochem 1997; 245: 64-70.

12 Fukui K, Takatsu H, Koike T, Urano S. Hydrogen peroxide induces neurite degeneration: prevention by tocotrienols. Free Radic Res 2011; 45: 681-691.

13 Kwon SH, Kim MJ, Ma SX, et al. Eucommia ulmoides Oliv. Bark. protects against hydrogen peroxide-induced neuronal cell death in SH-SY5Y cells. $J$ Ethnopharmacol 2012; 142: 337-345.

14 Jang JH, Surh YJ. Protective effects of resveratrol on hydrogen peroxideinduced apoptosis in rat pheochromocytoma (PC12) cells. Mutat Res 2001; 496: $181-190$.

15 Ginn-Pease ME, Whisler RL. Redox signals and NF- $\kappa \mathrm{B}$ activation in T cells. Free Radic Biol Med 1998; 25: 346-361.

16 Finkel T. Oxygen radicals and signaling. Curr Opin Cell Biol 1998; 10: 248 253.

17 Gao Y, Chu SF, Li JP, et al. Do glial cells play an anti-oxidative role in Huntington's disease? Free Radic Res 2014; 48: 1135-1144.

18 Gardner AM, Xu FH, Fady C, et al. Apoptotic vs. nonapoptotic cytotoxicity induced by hydrogen peroxide. Free Radic Biol Med 1997; 22: 73-83.

19 Chen Q, Ames BN. Senescence-like growth arrest induced by hydrogen peroxide in human diploid fibroblast F65 cells. Proc Natl Acad Sci US A 1994; 91: 4130-4134.

20 Schraufstatter IU, Hinshaw DB, Hyslop PA, Spragg RG, Cochrane CG. Oxidant injury of cells. DNA strand-breaks activate polyadenosine diphosphateribose polymerase and lead to depletion of nicotinamide adenine dinucleotide. J Clin Invest 1986; 77: 1312-1320.

21 Fukui K, Ushiki K, Takatsu H, Koike T, Urano S. Tocotrienols prevent hydrogen peroxide-induced axon and dendrite degeneration in cerebellar granule cells. Free Radic Res 2012; 46: 184-193.

22 Fukui K, Sekiguchi H, Takatsu H, Koike T, Koike T, Urano S. Tocotrienol prevents AAPH-induced neurite degeneration in neuro2a cells. Redox Rep 2013; 18: 238-244.

23 Coleman M. Axon degeneration mechanisms: commonality amid diversity. Nat Rev Neurosci 2005; 6: 889-898.

24 Errea O, Moreno B, Gonzalez-Franquesa A, Garcia-Roves PM, Villoslada P. The disruption of mitochondrial axonal transport is an early event in neuroinflammation. J Neuroinflammation 2015; 12: 152.

25 Gundimeda U, McNeill TH, Barseghian BA, et al. Polyphenols from green tea prevent antineuritogenic action of Nogo-A via $67-\mathrm{kDa}$ laminin receptor and hydrogen peroxide. J Neurochem $2015 ; 132$ : 70-84.

26 Podratz JL, Windebank AJ. NGF rescues DRG neurons in vitro from oxidative damage produced by hemodialyzers. Neurotoxicology 2005; 26: 343-350.

27 Song JH, Miyazawa T. Enhanced level of n-3 fatty acid in membrane phospholipids induces lipid peroxidation in rats fed dietary docosahexaenoic acid. Atherosclerosis 2001; 155: 9-18.

28 Fukuzawa K. Dynamics of lipid peroxidation and antioxidation of alphatocopherol in membranes. J Nutr Sci Vitaminol (Tokyo) 2008; 54: 273-285.

29 Sensi SL, Yin HZ, Carriedo SG, Rao SS, Weiss JH. Preferential $\mathrm{Zn}^{2+}$ influx through $\mathrm{Ca}^{2+}$-permeable AMPA/kainate channels triggers prolonged mitochondrial superoxide production. Proc Natl Acad Sci US A 1999; 96: 24142419.

30 Lee YS, Lee SJ, Seo KW, Bae JU, Park SY, Kim CD. Homocysteine induces COS-2 expression in macrophages through ROS generated by NMDA receptor-calcium signaling pathways. Free Radic Res 2013; 47: 422-431.

31 Colle D, Hartwig JM, Soares FA, Farina M. Probucol modulates oxidative stress and excitotoxicity in Huntington's disease models in vitro. Brain Res Bull 2012; 87: 397-405.

32 Suzuki M, Nagai M, Wada K, Koike T. Calcium leak through ryanodine receptor is involved in neuronal death induced by mutant huntingtin. Biochem Biophys Res Commun 2012; 429: 18-23.

33 Ikegami K, Koike T. Non-apoptotic neurite degeneration in apoptotic neuronal death: pivotal role of mitochondrial function in neurites. Neuroscience 2003; 122: 617-626.

34 Alim MA, Hossain MS, Arima K, et al. Tubulin seeds alpha-synuclein fibril formation. J Biol Chem 2002; 277: 2112-2117.

35 Cappelletti G, Surrey T, Maci R. The parkinsonism producing neurotoxin MPP+ affects microtubule dynamics by acting as a destabilising factor. FEBS Lett 2005; 579: 4781-4786.

36 Yang Y, Fukui K, Koike T, Zheng X. Induction of autophagy in neurite degeneration of mouse superior cervical ganglion neurons. Eur J Neurosci 2007; 26: 2979-2988.

37 Gerdts J, Summers DW, Milbrandt J, DiAntonio A. Axon self-destruction: new links among SARM1, MAPKs, and NAD+ metabolism. Neuron 2016; 89: 449-460.

38 Miller RH, Lasek RJ. Cross-bridges mediate anterograde and retrograde vesicle transport along microtubules in squid axoplasm. J Cell Biol 1985; 101: 2181-2193.

39 Fang W, Gao G, Zhao H, et al. Role of the Akt/GSK-3 $\beta /$ CRMP-2 pathway in axon degeneration of dopaminergic neurons resulting from MPP+ toxicity. Brain Res 2015; 1602: 9-19.

40 Lunn ER, Perry VH, Brown MC, Rosen H, Gordon S. Absence of wallerian degeneration does not hinder regeneration in peripheral nerve. Eur J Neurosci 1989; 1: 27-33.

41 Press C, Milbrandt J. Nmnat delays axonal degeneration caused by mitochondrial and oxidative stress. J Neurosci 2008; 28: 4861-4871.

42 Moutal A, François-Moutal L, Perez-Miller S, et al. (S)-lacosamide binding to collapsin response mediator protein 2 (CRMP2) regulates CaV2.2 activity by subverting its phosphorylation by cdk5. Mol Neurobiol 2016; 53: 1959 1976.

43 Miyasaka T, Morishima-Kawashima M, Ravid R, et al. Molecular analysis of mutant and wild-type tau deposited in the brain affected by the FTDP-17 R406W mutation. Am J Pathol 2001; 158: 373-379.

44 Nishimura T, Fukata Y, Kato K, et al. CRMP-2 regulates polarized Numbmediated endocytosis for axon growth. Nat Cell Biol 2003; 5: 819-826.

45 Touma E, Kato S, Fukui K, Koike T. Calpain-mediated cleavage of collapsin response mediator protein (CRMP)-2 during neurite degeneration in mice. Eur J Neurosci 2007; 26: 3368-3381.

46 Fukui K, Masuda A, Hosono A, et al. Changes in microtubule-related proteins and autophagy in long-term vitamin E-deficient mice. Free Radic Res 2014; 48: 649-658

47 Fukui K, Kawakami H, Honjo T, et al. Vitamin E deficiency induces axonal degeneration in mouse hippocampal neurons. J Nutr Sci Vitaminol (Tokyo) 2012; 58: 377-383.

48 Cho Y, Park D, Cavalli V. Filamin A is required in injured axons for HDAC5 activity and axon regeneration. J Biol Chem 2015; 290: 22759-22770.

49 Cho Y, Cavalli V. HDAC5 is a novel injury-regulated tubulin deacetylase controlling axon regeneration. EMBO J 2012; 31: 3063-3078.

50 Cappelletti G, Maggioni MG, Ronchi C, Maci R, Tedeschi G. Protein tyrosine nitration is associated with cold- and drug-resistant microtubules in neuronal-like PC-12 cells. Neurosci Lett 2006; 401: 159-164.

51 Stokin GB, Lillo C, Falzone TL, et al. Axonopathy and transport deficits early in the pathogenesis of Alzheimer's disease. Science 2005; 307: 1282-1288.

52 Li H, Li SH, Yu ZX, Shelbourne P, Li XJ. Huntingtin aggregate-associated axonal degeneration is an early pathological event in Huntington's disease mice. J Neurosci 2001; 21: 8473-8481.

53 Lin WJ, Kuang HY. Oxidative stress induces autophagy in response to multiple noxious stimuli in retinal ganglion cells. Autophagy 2014; 10: 1692-1701.

54 Høyer-Hansen M, Bastholm L, Szyniarowski P, et al. Control of macroautophagy by calcium, calmodulin-dependent kinase kinase-beta, and Bcl-2. Mol Cell 2007; 25: 193-205.

55 Praprotnik D, Smith MA, Richey PL, Vinters HV, Perry G. Filament heterogeneity within the dystrophic neurites of senile plaques suggests blockage of fast axonal transport in Alzheimer's disease. Acta Neuropathol 1996; 91: 226-235.

56 Cole AR, Noble W, van Aalten L, et al. Collapsin response mediator protein2 hyperphosphorylation is an early event in Alzheimer's disease progression. J Neurochem 2007; 103: 1132-1144.

57 Petratos S, Li QX, George AJ, et al. The beta-amyloid protein of Alzheimer's disease increases neuronal CRMP-2 phosphorylation by a Rho-GTP mechanism. Brain 2008; 131(Pt 1): 90-108.

58 Bogdanov MB, Ferrante RJ, Kuemmerle S, Klivenyi P, Beal MF. Increased vulnerability to 3-nitropropionic acid in an animal model of Huntington's disease. J Neurochem 1998; 71: 2642-2644.

59 Ribeiro M, Rosenstock TR, Oliveira AM, Oliveira CR, Rego AC. Insulin and IGF-1 improve mitochondrial function in a PI-3K/Akt-dependent manner and reduce mitochondrial generation of reactive oxygen species in Huntington's disease knock-in striatal cells. Free Radic Biol Med 2014; 74: 129-144.

60 Fischer LR, Culver DG, Tennant P, et al. Amyotrophic lateral sclerosis is a distal axonopathy: evidence in mice and man. Exp Neurol 2004; 185: 232-240.

61 Frei B, Richter C. N-methyl-4-phenylpyridine $\left(\mathrm{MMP}^{+}\right)$together with 6-hydroxydopamine or dopamine stimulates $\mathrm{Ca}^{2+}$ release from mitochondria. 
FEBS Lett 1986; 198: 99-102.

$62 \mathrm{Lu} \mathrm{X,} \mathrm{Kim-Han} \mathrm{JS,} \mathrm{Harmon} \mathrm{S,} \mathrm{Sakiyama-Elbert} \mathrm{SE,} \mathrm{O'Malley} \mathrm{KL.} \mathrm{The}$ parkisonian mimetic, 6-OHDA, impairs axonal transport in dopaminergic axons. Mol Neurodegener 2014; 9: 17.

63 Glinka YY, Youdim MB. Inhibition of mitochondrial complexes I and IV by 6-hydroxydopamine. Eur J Pharmacol 1995; 292: 329-332.
64 Sano M, Ernesto C, Thomas RG, et al. A controlled trial of selegiline, alphatocopherol, or both as treatment for Alzheimer's disease. The Alzheimer's Disease Cooperative Study. N Engl J Med 1997; 336: 1216-1222.

65 Cox CJ, Choudhry F, Peacey E, et al. Dietary (-)-epicatechin as a potent inhibitor of $\beta \gamma$-secretase amyloid precursor protein processing. Neurobiol Aging 2015; 36: 178-187. 